\title{
17 \\ Unlucky End: On the Obsolescence of Products and Books
}

As this book was being written, BVMF decided to allow more open contracts in its interest rate futures, not just every 3 months. It also changed almost all of the details of its inflation-linked futures.

The relationship between the CDI and the Selic rate has also changed. Since the end of 2013, the number of contributions to the formation of the CDI rate dropped dramatically (the incentives to contributing to the formation of benchmark rates are lower than the costs of implementing a whole governance policy around it); because of that CETIP has implemented a fallback that models the CDI using a linear regression with the Selic rate as the independent variable (not the best available choice, in our opinion). The consequences of choosing such a model are left to the reader.

Brazil being Brazil, the only thing we can be sure about is that we cannot be sure about anything else. If we had written this book 5 years ago, we would have missed a lot of developments like the IOF on Derivatives or the CDI fallback. 10 years ago we would be heralding a new era, oblivious to what would happen in 2008. We will continue to monitor relevant events on taxes, convertibility, market and regulatory changes, and will keep improving and updating models. Please visit the book's website to keep yourself informed of these developments, of upcoming new editions, of interesting snippets of code and analysis.

Books on financial markets will by its nature become obsolete. We hope to have written a book that, rather than just list facts, will have increased your interest in Brazil's financial markets and encouraged you to develop your own solutions and models. If we have helped you to avoid obsolescence, our goal will have been achieved. 